\title{
IRMA: a Graphical Tool for Interplanetary Mission Design
}

\author{
Giancarlo Genta and P. Federica Maffione, \\ DIMEAS, Politecnico di Torino, Torino, Italy
}

\begin{abstract}
Designing an interplanetary mission is a complex task and requires the choice of the launch opportunity and of the exact launch and arrival dates. Depending on these choices, the trajectory must be defined and, in case of continuous thrust, also the thrust profile needs to be optimized..

Traditionally, these choices are made using some plots which allow to find a good compromise between the travel duration and the cost of the mission, which is often expressed in terms of initial mass in Earth orbit (IMLEO). IRMA (InterPlanetary Mission Analysis) code, based on the MATLAB ${ }^{\circledR}$ environment, is here described. It allows to deal with both impulsive propulsion (using the patched conics approach) and low continuous thrust (Solar or Nuclear electric or propellantless, like solar sails). A specific solver, based on indirect optimization techniques, has been developed specifically for this program, but IRMA can be used also as an interface for standard solvers, based on direct methods, like the FALCON.m code.
\end{abstract}

\section{Introduction}

The planets of our Solar System move in a complex way around the Sun, and this causes the performances of any space mission to depend on the launch opportunity and, once the latter has been chosen, on the exact launch date.

The precision required in the computation of the spacecraft trajectory is such that the approximation obtained assuming that the planets move along circular and even elliptical orbits is not sufficient for the analysis of an interplanetary mission, and the knowledge of detailed ephemerides is required. While historically ephemerides consisted in tables where the positions of the celestial bodies of interest were reported as functions of time, modern ephemerides are based on mathematical models allowing to compute the position of the solar system bodies at any given time. Precise reference frames and time reference must then be stated: at present the ephemerides made available by NASA-JPL $[1,2]$ are based on the International Celestial Reference Frame (ICRF) and on the Julian time.

The ICRF is an inertial reference frame centred in the centre of mass of the solar system and whose axes are fixed to a set of 295 extra-galactic radio sources. The axes directions are assumed to be very close to those of the axes of the Earth-fixed J2000 frame, and so its $x$-axis points in the direction of the vernal equinox and the $y$ axis is contained in the Earth equatorial plane in its position at 12.00 of January 1st, 2000. For solar system navigation it is perhaps better to rotate the reference frame about its $x$-axis of the obliquity angle $\varepsilon$ of the Earth axis, so that the $x-y$ plane coincides with the ecliptic plane. An Earth-fixed reference frame with the axes so oriented is the ECLIPJ2000 frame.

The solar system is particularly simple, with respect to the many star systems in which the central star is multiple; moreover the mass of the Sun is much greater, by orders of magnitude, than that of all other bodies. As a consequence, the trajectories of all bodies, natural and artificial, are very close to conic sections and, in particular, to ellipses.

Strictly speaking, the motion of a spacecraft in the solar system is an $n$-bodies restricted problem, since not only the Sun, but also all the planets and the other bodies affect the trajectory of the spacecraft. The term 'restricted' is used to state that while the mass of the Sun and of the planets affect the trajectory of the spacecraft, the mass of the latter doesn't affect the motion of the Sun and of the planets. In the restricted problem the positions of the celestial bodies is known and obtained from the ephemerides, and from them the trajectory of the spacecraft is obtained.

In many cases it is not enough to account for the gravitational attraction of the Sun and the planets, but also the pressure of the Sun light on the spacecraft must be accounted for.

When a spacecraft is close to a planet, it is possible to assume that its trajectory is affected only by the attraction of the planet and the Sun, and then the socalled restricted 3 bodies problem (the 3 bodes are the Sun, the planet and the spacecraft) can be used, which, by the way, has no closed form solution.

From the restricted 3 body problem it is possible to derive two important concepts: the sphere of influence, and the libration points.

The sphere of influence of a body orbiting around another one (usually the mass of the first is much smaller

Corresponding author: giancarlo.genta@polito.it 
than the mass of the second one ${ }^{a}$ ) is the zone of Space in which the motion of a third body is much more affected by the mass of the small body (planet) than that of the large one (sun). It is not really a sphere, although it is possible to approximate it in that way as a first approximation.

The libration points (or Lagrange points) are locations in space in which the gravitational accelerations of the two bodies and the centripetal acceleration due to the rotation about the 'large' body are in equilibrium so that a third body can remain in that point. There are 5 libration points for each sun-planet pair. The equilibrium in some libration point is stable, that in other points is not, but all of them can be used to locate a spacecraft or a space station (if it is unstable, the spacecraft can be located in a halo orbit) like the case of the DSG (Deep Space Gateway, now Lunar Orbital Platform-Gateway) which will be located in the L4 point of the Earth-Moon system.

An interplanetary travel can thus be subdivided into 3 parts and in each one of them the spacecraft can be assumed to move only under the influence of a single celestial body, i.e. each arc of the trajectory can be studied using the restricted two body problem.

A spacecraft leaving planet A and aimed to planet B, first moves in the sphere of influence of planet A (under the effect of the attraction of planet $\mathrm{A}$ ), then moves outside the spheres of influence of all planets, under the effect of the Sun, and then enters the sphere of influence of planet $\mathrm{B}$, under the effect of the attraction of planet $\mathrm{B}$ alone.

The trajectory can thus be approximated by 3 arcs (patched arcs approach), which in general must be computed numerically.

Assume first that the spacecraft is propelled by a high thrust or impulsive system, i.e. a thruster which supplies a thrust much higher than all other forces acting on the spacecraft for a time which is much shorter that the travel time. The thrust is assumed to be similar to a Dirac's delta, i.e. an infinitely large force applied for an infinitesimal time, so that it results in an instant velocity variation $\Delta V$ and the trajectory is determined by the gravitational attractions only (coasting arcs). Moreover, each coasting arc is approximated by an arc of a conical section determined by the attraction of a single celestial body, that in whose sphere of influence the spacecraft lies, i.e. that exerting the largest gravitational acceleration., The 3 arcs are parts of conical sections (circles, ellipses, parabolas or hyperbolas), and this approximation is called the patched conics approach.

The three arcs are usually hyperbolic for the parts within the two planetary spheres of influence and elliptical for the heliocentric parts.

The thrust bursts are best applied at the beginning and at the end of the journey (in the latter case aerodynamic forces due to the atmosphere of the destination planet can be used), but usually the conic

a For instance, the mass of the Earth is about $3 \times 10^{-6}$ times the mass of the Sun and the mass of the Moon is about 0.0123 the mass of the Earth. arcs are interrupted by (usually small) velocity variations aimed at correcting the overall trajectory. More complex trajectories can be split in a larger number of arcs, like for instance when a gravity assist manoeuvre is performed in the sphere of influence of another planet.

In case of low thrust systems, i.e. the thrust is not much higher than the other forces acting on the spacecraft and is applied for long times, possibly up to the whole travel time, the same scheme can be applied by subdividing the trajectory in arcs performed in the sphere of influence of a single body. The propelled arcs are, in this case, no more parts of conical sections, looking like arcs of spirals, which are neither logarithmic nor Archimedes spirals, and cannot be computed in closed form, so that the numerical integration of the trajectory becomes mandatory.

This patched arcs approach is thus the simplest, although approximate, way of computing the trajectory of a spacecraft in the solar system ${ }^{\mathrm{b}}$. It is generally applied for the early phases of the design of interplanetary missions, when the departure and arrival dates are chosen and a vey large number of trajectories (tens or hundreds of thousands) must be computed. For a fine tuning of the mission, later, more precise approaches, based on the restricted n-boy problem, must be used.

There are however cases in which the patched arcs approach cannot be used even in the early phase of the mission, namely when instead of true interplanetary missions, missions between a planet and its satellite(s) is considered. This was the case of the Apollo missions, or of some missions in the system of the Jupiter's or Saturn's satellites. The Earth-Moon trajectories cannot be computed using the patched conics approach, since in a large part of the trajectory the motion of the spacecraft is affected by the attraction of both bodies, and the same is true for the trajectories to reach the libration points. The gravitational field in the Earth-Moon system is quite complex, and even more complex is the gravitational field in the system of Jupiter (or Saturn) and its many satellites.

This cannot be neglected even in the early phases of the design of mission, as it was the case of the Apollo missions, which followed trajectories shaped as a figure 8 about the two celestial bodies. A long trial and error work was performed at that time, but even today is not easy to design algorithms to obtain these trajectories.

\section{The choice of the launch date}

\footnotetext{
${ }^{b}$ It must be expressly stated that this approach will be impossible when the problem of travelling in the system around a multiple star will be faced. If a mission like that studied in the Breakthrough Starshot project will aim to perform more than just a flyby in the Alpha Centaury system, even its preliminary study will need to go beyond the patched arcs assumption.
} 
After deciding the launch opportunity - which is usually characterized by an opposition of the planet (the two planets and the Sun are almost in a straight line, with the two planets at their smallest distance) - the exact launch date must be decided. In the IRMA code this is done by plotting a suitable diagram.

The problem of choosing the best start and arrival date can be mathematically stated as the search of the values of the start and the arrival dates $\left(T_{s}\right.$ and $\left.T_{a}\right)$ or, which is the same, the start date and the travel duration $\left(T_{s}\right.$ and $T$ ) which yield the minimum value of a cost function I. Depending on the propulsion type and the mission goals, different formulations of the cost function $I$ can be chosen.

For instance, in case of an interplanetary travel starting from Low Earth Orbit (LEO) with the aim of bringing cargo to a planet, say Mars, the cost function may be the Initial Mass in LEO (IMLEO), which is linked with the cost of the mission. In case of a human mission to the same planet, the IMLEO is not the only important parameter, since the duration $T$ of the travel is in itself a parameter to be minimized and a compromise between $T$ and the IMLEO must be looked for.

Another important difference is whether the mission under consideration is a one-way mission, e.g. launching a probe, delivering a cargo or performing a one-way colonization mission, or a two-way mission like a human exploration mission. In this case the parameters to be chosen are the starting and arrival dates of the outbound and inbound journeys $\left(T_{s o}, T_{a o}, T_{s i}\right.$ and $\left.T_{a i}\right)$ or the overall starting date, the duration of both journeys and the stay on the planet $\left(T_{s o}, T_{o}, T_{p}\right.$ and $\left.T_{i}\right)$.

Consider the optimization of a one-way journey: the problem is to find the values of $T_{s}$ and $T_{a}$ (or of $T_{s}$ and $T$ ) yielding the minimum of function $I\left(T_{s}, T_{a}\right.$ ) (or of function $I\left(T_{s}, T\right)$ ) - if such a minimum exists - or yielding a suitable compromise between minimizing $I$ and minimizing $T$.

To solve this problem the value of $I$ is computed for a large number of missions characterized by different values of $T_{s}$ and $T_{a}$ and the surface $I\left(T_{s}, T_{a}\right)$ is plotted, in particular in the form of a contour plot. The fact that a minimum of the cost function may not exist, or may lead to an unacceptably long travel time, justifies the computationally heavy approach mentioned above instead of using more efficient optimization methods like those based on a gradient descent. What is essential in this case is to gain a good knowledge of the overall shape of the surface $I\left(T_{s}, T_{a}\right)$ to search for an adequate compromise between low cost function and low travel time.

The cost function can be computed for the interplanetary journey only, or function $I\left(T_{s}, T_{a}\right)$ can be defined to take into account the whole journey, from the starting orbit around the first planet (or the planetary surface) to the arrival orbit about the second planet. In this case the plot can be obtained only after many details of the mission have been stated (starting and arrival orbits parameters, possible flybys, aerobraking manoeuvres, trajectory corrections, etc.).

The computation of the plot is quite computationally intensive, since it requires to compute thousands (possibly hundred thousand or millions) trajectories. Each one of the trajectories may involve an optimization procedure, particularly in the case of low thrust, in which case not only the trajectory, but also the thrust profile, needs to be computed. However, the choice of the launch and arrival dates can be performed in a few hours using a laptop computer.

\subsection{Impulsive Thrust}

The tool traditionally used for one-way impulsive missions is the so-called pork-chop plot. It has been used since the 1970s, when it was introduced for the Voyager program, $[3,10]$. The classical pork-chop plot uses as cost function the square of the hyperbolic excess speed $C_{3}=V_{\infty}{ }^{2}$ as a function of $T_{s}$ and $T_{a}$ and thus takes into account only the energy of the trajectory when leaving the starting planet's sphere of influence.

If the planetary orbits were circular and coplanar, the surface $C_{3}\left(T_{s}, T_{a}\right)$ would have a single minimum corresponding to the Hohmann transfer, which in the case of Earth-Mars trajectories starts about 96 days before the opposition and has a duration of 258.73 days. Since in an actual case the orbits of the planets must be considered at least elliptical and not coplanar, the porkchop plot depends on the specific launch opportunity and usually the surface $C_{3}\left(T_{s}, T_{a}\right)$ has two minima, one related to Type I missions (trajectories which intersect the orbit of the arrival planet the first time at the arrival point) and the other to Type II missions (trajectories intersecting the orbit of the arrival planet once before the arrival time).

The pork-chop plot is usually plotted for a limited range of starting dates around the optimal launch window, but it may be extended indefinitely to study what happens also in case of a launch well outside the optimal conditions. This may seem to have very little practical interest, but it may be useful for designing short-stay missions or if an advanced propulsion system - e. g. Nuclear Thermal (NTP) - allows to perform a mission to deliver badly needed cargo to an outpost or to allow the return of personnel in an emergency.

It is also possible to compute the total orbit-to-orbit $\Delta V$, i.e. the sum of the velocity increments required to enter the interplanetary trajectory from the parking orbit plus that required to enter the orbit about the arrival planet from the former. In case the manoeuvres at the arrival planet are performed using the aerodynamic drag of the planetary atmosphere (aerobraking or aerocapture manoeuvres), the second $\Delta V$ is set to 0 or to a low value. Such a plot can be defined as a $\Delta V$-plot.

As an example, the $\Delta V$-plot for Earth-Mars transfers performed between mid-August 2034 (400 days before the 2035 opposition) and the end of December 2038 (1200 after the same opposition), is shown in Fig. 1. The departure orbit is circular and has an altitude of $900 \mathrm{~km}$ (a possible choice for NTP), while the arrival orbit is highly elliptical with a periareion of $400 \mathrm{~km}$ and an apoareion of $30,000 \mathrm{~km}$.

The dotted lines are the arrival dates, expressed in days after the 2035 opposition. 
The plot covers more than two complete synodic periods, and the differences between the two launch opportunities are clear. In the 2035 opportunity the optimal launch window is around 80 days before opposition and the minimum energy trajectory is of Type
I with a duration of about 200 days. A good choice is launching on June 27, 2035, which requires $\Delta V_{1}=3,542$ $\mathrm{m} / \mathrm{s}$ and $\Delta V_{2}=921 \mathrm{~m} / \mathrm{s}$, with a total $\Delta V$ of $4,468 \mathrm{~m} / \mathrm{s}$.

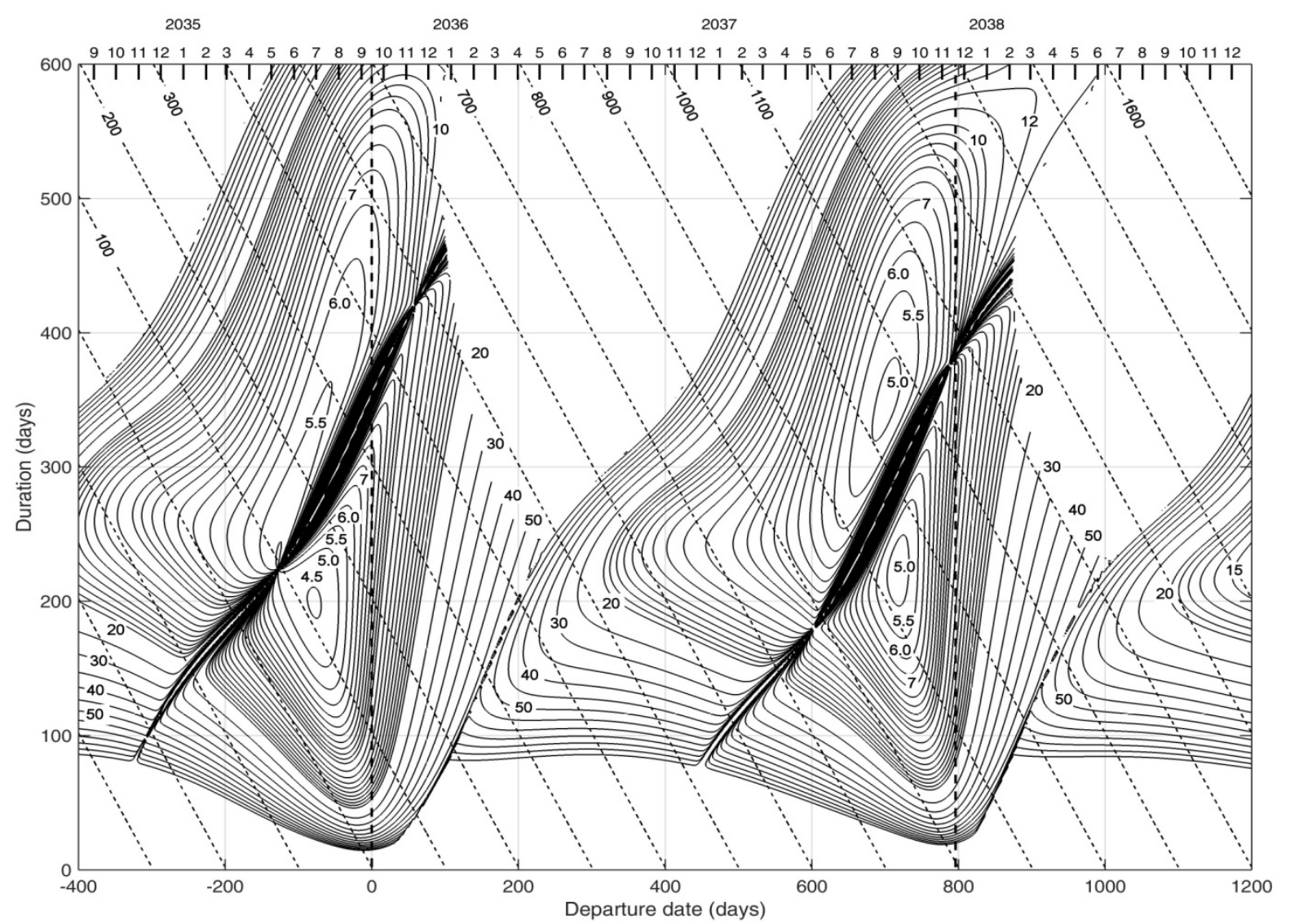

Fig. 1. $\Delta V$-plot for Earth-Mars transfers performed between mid-August 2034 and the end of December 2038. The $\Delta V$ is expressed in $\mathrm{km} / \mathrm{s}$ and the time is in days.

The plot was obtained using the IRMA code, with a routine based on the Newton Raphson method [11], solving the Gauss problem and the ephemeris data downloaded from the Jet Propulsion Laboratory's online resources [12]. The total number of trajectories computed is 240,000 .

\subsection{Low Thrust}

The case of low thrust is increasingly more important, since a number of robotic planetary missions are propelled by electric thrusters - mostly ion thrusters - $[13,14]$ powered by solar arrays (Solar Electric Propulsion, SEP), often in conjunction with gravity assist manoeuvres, and in the future Nuclear Electric Propulsion is much promising [15]. Since the early studies of human Mars missions, the possibility of using electric propulsion (mostly NEP, but also SEP) was considered $[16,17]$. In this case the time spent in space becomes a very important parameter of the mission, and the use of gravity assist manoeuvres is seldom considered, except for performing missions which would be impossible without exploiting the gravitational field of a planet different from the departure or arrival planets (in case of Mars missions, the Venus' gravitational field).

A plot similar to the pork-chop or the $\Delta V$-plot, but for low, continuous thrust, trajectories which can be applied to NEP and SEP spacecrafts, usually referred to as the bacon plot, has recently been introduced [18]. In this case the useful mass carried to the destination planet (payload plus structures) $m_{L}+m_{s}$, obviously at equal initial mass $m_{i}$ is assumed as a cost function to be maximized instead of minimized. Better, what is to be maximized is the ratio $\left(m_{L+} m_{s}\right) / m_{i}$. To use a true cost function to be minimized, its reciprocal can be used: $I=$ $m_{i} /\left(m_{L}+m_{s}\right)$.

The first difference between the pork-chop plot and the bacon plot is related to the mathematical approach used to compute them. While in case of impulsive mission the patched conics approach can be used and the trajectory can be obtained in closed form, in case of low thrust mission the trajectory must be computed together with the thrust profile and different types of optimization techniques, both direct or indirect, can be used [19]. The second difference is that in case of low thrust the contours are not closed, i.e. the cost function $I$ has no 
minima and the longer is the travel time, the lower is the value of the cost function.

In case of low-thrust mission, it is also necessary to distinguish between Variable Ejection Velocity (VEV) and Constant Ejection Velocity (CEV) systems [20-23]. The simplest and most convenient approach is assuming that the system operates at constant power (in the case of NEP) or at the maximum power allowed by the solar arrays (in the case of SEP) [24 - 27] and when the computed thrust profile requires a reduction of the thrust, this is obtained by increasing the specific impulse. This approach is described in detail in $[28,29]$ and leads to much less computationally intensive algorithms. However, it has a basic limitation: the maximum value of the specific impulse which is required, in particular in the middle of the interplanetary cruise, is much higher than the maximum value the thruster can actually reach, even if particularly suitable plasma thrusters, like the $\operatorname{VASIMR}^{\circledR}[15,30,31]$, are used.

When the thrust must be reduced below the value corresponding to the maximum specific impulse, the simplest action is to keep the latter at the maximum possible value and to reduce the power, but this leads to a non-optimal control law and then to an increase of the cost function. A much better alternative is maintaining the power at its maximum possible value when the thruster is on, and switching off the thruster, for a certain time. The engine on-off times are among the parameters to be optimized, together with the thrust direction and intensity and the specific impulse.

The bacon plot can be obtained using both direct or indirect optimization methods. Of course also stochastic methods can be used but, since a large number of trajectories must be computed and the problem is a constrained one, they become too computationally intensive and for this reason are not considered here. The IRMA routines are based on the approach proposed by Keaton in [28] and the boundary value problem is solved by using the BVP5C [32] MATLAB ${ }^{\circledR}$ routine based on the four-stage Lobatto IIIa formula and implemented as an implicit Runge-Kutta formula.

If direct methods are considered, IRMA uses the optimal control software Falcon.m, developed at the Institute of Flight System Dynamics of Munich [33]. The problem is converted into a nonlinear programming problem using the trapezoidal transcription method and then solved using the numerical solver IPOPT (Interior Point OPTimizer) [34]. A more detailed discussion of the solution techniques can be found in $[35,36]$.

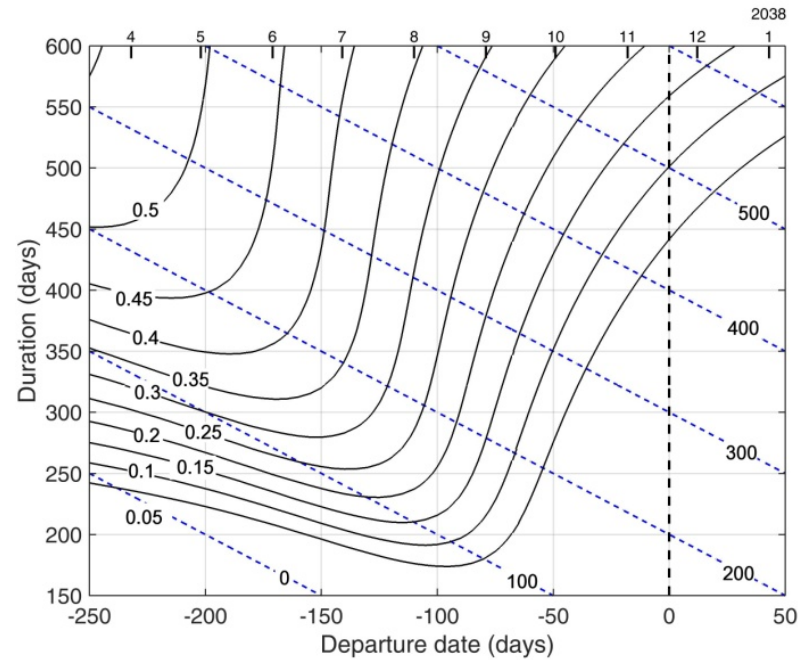

Fig.2. Orbit-to orbit bacon plot for NEP for Earth-Mars travels in the launch opportunity of 2037. Computation performed using the IRMA code.

Also in the case of low thrust it is possible to obtain an orbit-to-orbit plot. However, while in the case of impulsive thrust, the duration of the first and the last phase, performed within the spheres of influence of the start and destination planets, can be neglected, in the present case they can take a substantial fraction of the total time. The relative durations of the three phases constitute a set of parameters which are essential in the overall optimization of each mission.

An orbit-to-orbit bacon plot computed using IRMA for Earth-Mars travels in the launch opportunity of 2037, with $\alpha=10 \mathrm{~kg} / \mathrm{kW}$, maximum specific impulse $I_{\text {smax }}=$ $7000 \mathrm{~s}$, thrusters efficiency of $65 \%$ and tankage factor of 0.06 is reported in Fig. 2. The starting and arrival orbits are both circular, with an height of $800 \mathrm{~km}$ and $300 \mathrm{~km}$ respectively. The plot has been obtained by using the ephemerides developed by JPL [2].

A possible choice for a crew ship is a starting time of 80 days before opposition, and a travel duration of 210 days, which yield. $\left(m_{L}+m_{s}\right) / m_{i}=0.1511$.

\subsection{Propellantless propulsion}

The possibility of travelling in space without the need of propellant is extremely interesting. At present the only devices which allow to produce a thrust without the need of expelling a propellant are solar sails. They produce very small thrust, but one which may be adequate for a number of robotic missions. With further technological improvements aimed to build very low mass sails, there is the possibility of extending their applicability to other types of missions.

Apart from solar sails, propelled by solar light, there is the possibility of using laser sails ${ }^{\mathrm{c}}$ and magnetic sails, propelled by solar wind. Still hypothetical (and much controversial) is the possibility of future propellantless

\footnotetext{
${ }^{\mathrm{c}}$ Laser sails have been proposed also for interstellar journeys (see, for instance [38]) and are at the base of the Breakthrough Starshot project [39]. Solar and laser sails are collectively referred to as light sails.
} 
propulsion systems like those studied in the NASA Breakthrough Propulsion Physics (BPP) project [37].

In all these systems it is possible to define a specific thrust, i.e. a ratio between the thrust supplied by the propulsion system and its total mass. In the case of solar sails the specific thrust decreases with the square of the distance from the Sun. In case of sails of all types, the value of the thrust depends of the orientation of the sail, reducing to zero when the sail is in a plane containing the light source. Each trajectory, characterized by a starting and an arrival date, can be optimized by searching the direction of the thrust which minimizes the total mass of the propulsion system (or better the ratio between the propulsion system and the total mass of the spacecraft $\left.m_{p s} / m_{i}\right)$ [26].

A plot of $I=m_{p s} / m_{i}\left(T_{s}, T_{a}\right)$ can thus be obtained also in the case of propellantless systems, although this plot does not yet have a name.

\subsection{Two ways journeys}

As already stated, a two-ways mission can be optimized as a whole; in particular this is the case for human Mars missions when the two journeys are performed in the same launch opportunity (short stay missions). Also in this case the optimal dates can be obtained by plotting a suitable contour plot. Such plots, obtained through the IRMA code, are described in detail in $[40,41]$

\section{SIngle mission computation}

\subsection{Impulsive propulsion}

Once the starting and arrival dates have been stated, it is easy to design the mission. In the IRMA code, it is possible to chose to use the patched conics approach or not. In the first case, as stated in session 2.1, the Gauss problem is solved using the Newton Raphson method [11]. The trajectory can be assumed to start outside the sphere of influence of the planet, i.e. at a distance from is centre equal to

$$
d=r_{p}\left(\frac{m_{p}}{m_{s}}\right)^{2 / 5} \frac{1}{\sqrt[10]{1+3 \cos ^{2}(\theta)}}
$$

where $d$ is the distance from the planet, $r_{p}$ is the distance from the planet from the Sun, $m_{p}$ and $m_{s}$ are the masses of the two bodies and $\theta$ is the angle between the direction of at which the spacecraft is located at start and the direction of the Sun. In case of starting tangentially to the orbit- as it is the case for a Hohmann transfer $-\theta$ is close to $90^{\circ}$. Since at this point of the computation it is impossible to assess the correct value of $\theta$, and eq. (1) is little influenced by its value, in IRMA the term in $\theta$ is neglected.

The interplanetary trajectory can be easily computed through the equations in Cartesian coordinates:

$$
\left\{\begin{array}{l}
\dot{x}=v_{x} \\
\dot{y}=v_{y} \\
\dot{z}=v_{z} \\
\dot{v}_{x}=-\sum_{\forall i} \frac{\mu_{i}\left(x-x_{i}\right)}{\sqrt{\left[\left(x-x_{i}\right)^{2}+\left(y-y_{i}\right)^{2}+\left(z-z_{i}\right)^{2}\right]^{3}}} \\
\dot{v}_{y}=-\sum_{\forall i} \frac{\mu_{i}\left(y-y_{i}\right)}{\sqrt{\left[\left(x-x_{i}\right)^{2}+\left(y-y_{i}\right)^{2}+\left(z-z_{i}\right)^{2}\right]^{3}}} \\
\dot{v}_{z}=-\sum_{\forall i} \frac{\mu_{i}\left(z-z_{i}\right)}{\sqrt{\left[\left(x-x_{i}\right)^{2}+\left(y-y_{i}\right)^{2}+\left(z-z_{i}\right)^{2}\right]^{3}}}
\end{array}\right.
$$

where the sum is extended to all the attracting bodies considered and the initial and final conditions are stated on the positions (i.e. $x, y$, and $z$ are stated at the initial and final instants, while the components of the velocity at the same instants are among the unknowns and allow to compute the $\Delta \mathrm{V}$ at the starting and arrival planet).

The same equations can be written in cylindrical or spherical coordinates, and nondimensional coordinates can be used, to improve the convergence of the solution algorithm.

The solution of the Boundary Value Poblem (BVP) is performed using the already mentioned BVP5C MATLAB $^{\circledR}$ algorithm [32].

Operating in this way, it is possible to take into account the gravitational attraction of as many celestial bodies as required. The arcs of trajectories so obtained are not conics - although usually they are not much different from being such - and other effects, like the light pressure from the Sun, can be easily added at right hand side of the equations of motion. Clearly, the computer time is increased, but not of an unbearable amount.

\subsection{Low-thrust propulsion}

In case of low thrust propulsion (like NEP, SEP, solar sails or even more exotic, still to be developed, types) equations (2) can be modifes by simply writing at right hand side the cartesian components of the thrust vector $\mathbf{T}$ or, better, of the acceleration $\mathbf{a}=\mathbf{T} / m$, in which both $\mathbf{T}$ and $m$ are functions of time. In the case of electric propulsion, if the specific impulse is variable (either in an arbitrarily large range or, if limited, the nonoptimal strategy of reducing the power is chosen) equations (2) are easily modifies as

$$
\left\{\begin{array}{c}
\ddot{\mathbf{r}}=-\nabla U(\mathbf{r}, t)+\mathbf{a}(t) \\
\ddot{\mathbf{a}}=-\mathbf{a} \nabla[\nabla U(\mathbf{r}, t)]
\end{array}\right.
$$

Equation (3) can be expressed as a set of 12 first order equations in the position, velocity, acceleration and derivative of the acceleration. If an approach different from the restricted two body problem is used, the potential $U$ contains a sum (like in the last 3 equations 
(2) extended to all the bodies considered. It is also a known function of time, which is easily obtained from the ephemerides.

The boundary conditions are 12, the 6 initial and the 6 final components of the position and velocity. Actually, in this case the restricted $n$-body problem is not conceptually more complex then the restricted 2-body problem since even the latter requires the numerical integration of the trajectory (which is made of a sort of spiral, instead of conical sections, arcs.

Again, the solution is performed using the algorithm described in [32]. Accounting for more than one attracting celestial body - and possibly also of the pressure of the Sun light - does however increase (moderately) the computer time.

\subsection{Lunar missions}

Lunar exploration is again ranking quite high in the programs of both space agencies of several countries and private companies. As a consequence a new release of the IRMA code (IRMA 5.1) is presently being developed.

As already stated, navigation in the Earth-Moon system has some peculiarities, which make it more complex than travelling in the solar system in general, mainly owing to the ratio of the mass of the Moon with respect to that of Earth (0.0123) which is much higher than that of any planet with respect to the Sun (the highest is that of Jupiter, $9.55 \times 10^{-04}$ ): the Earth-Moon is almost a double planet, a thing which in the solar system occurs only for some dwarf planets and smaller bodies.

The study of lunar trajectories requires that at least the restricted 3 body problem is solved taking into account simultaneously the presence of Earth and Moon, and thus the trajectories are not conics. Since the Moon is within the sphere of influence of Earth (otherwise it would orbit the Sun and not Earth) the presence of the Sun and the orbiting of Earth about it can be neglected; however these effects can be accounted for if desired.

In the IRMA code a non-inertial reference frame is used: it is centred in the Earth-Moon centre of mass and rotates about it so that the $x$-axis lies on the Earth-Moon line. The equations of motion are modified to account for the resulting acceleration.

Since the algorithm to solve BVPs requires a starting solution, a trajectory obtained from the restricted two body problems about Earth (outside the sphere of influence of the Moon) and Moon (inside it) is first obtained: this trajectory is made of two arcs of ellipses for long transfer times, then the arc reaching the Moon becomes a hyperbola and for short transfer times both of them are hyperbolas. After computing the two arcs so that the velocities match when the sphere of influence of the Moon is entered, the two trajectories are rotated so that also the direction of the velocities match.

Trajectories of the type used for the Apollo missions are assumed: they cross the Earth-Moon line after entering the sphere of influence of the latter. Operating in this way the arrival orbit is retrograde with respect to the Moon rotation, which is not a problem since the Moon rotation is synchronous with it orbital motion, i.e. the Moon does not rotate in the reference frame used for the computation of the orbit.

One of such trajectories is shown in Fig. 3: a transfer time of 2.5 days was assumed and the starting and arrival orbits have heights of 300 and $200 \mathrm{~km}$ respectively. Since the Moon orbit is almost circular, the computations were computed under this assumption, but the correct Moon's ephemerides could be used.

The plane of the trajectory coincides with the Moon orbital plane, which is angled of just $5.14^{\circ}$ from the ecliptic, so it requires that the starting orbit has a suitable inclination with respect to the Earth's equator. This in turn sets limitations both on the possible launch site and on the timing of the mission..

In the plot both the initial trajectory, made of an ellipse about Earth (with $e=0.916$ ) and an hyperbola about the Moon (with $e=1.550$ ) and the final trajectory computed taking into account the presence of both The Earth and the Moon. From the figure it is clear that the two trajectories are quite similar, perhaps except for the last part within the sphere of influence of the latter. 


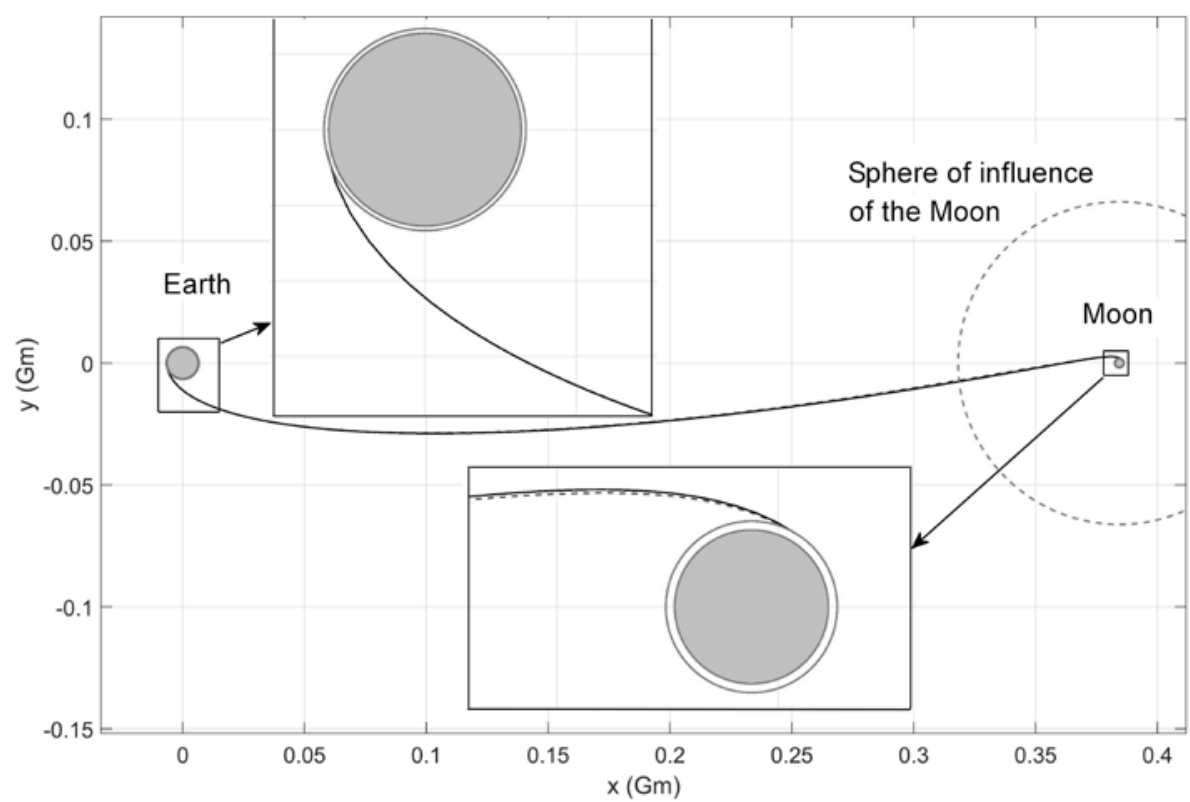

Fig.3. Trajectory for an impulsive lunar mission with a travel time of 2.5 days. A non-inertial frame centred in the centre of mass of the Earth-Moon system with $x$-axis on the Earth-Moon line is used. Both the starting approximation (elliptical and hyperbolic, dashed

line) and the numerical solution (full line) are shown. The starting and arrival orbits are at $300 \mathrm{~km}$ and $200 \mathrm{~km}$ from the surface.

The starting $\Delta \mathrm{V}$ is $3152 \mathrm{~m} / \mathrm{s}$, while at arrival a $\Delta \mathrm{V}$ of $860 \mathrm{~m} / \mathrm{s}$ is required. The total $\Delta \mathrm{V}$ of the mission is thus $4012 \mathrm{~m} / \mathrm{s}$.

\section{Conclusions}

The IRMA mission design code, based on the MATLAB $^{\circledR}$ environment, allows to study planetary missions performed in the solar system with different propulsion systems, using different choices for what the ephemerides of the various planets and the solution algorithms are concerned. The goals of the code are first providing general plots allowing to chose the starting date and the mission duration and then to study a single mission in detail. The first goal is performed using a patched arcs approach, based on the restricted two-body problem, while the second can include the gravitational perturbations due to the various planets and the pressure due to light of the Sun.

Owing to the importance that lunar exploration is again taking, a new version of the code is now being prepared to allow to study also lunar missions through at least the restricted 3-body problems.

The whole code is centred mostly on human missions so very slow missions based on multiple planetary flybys or lunar missions utilizing the fuzzy boundary dynamics are not included.

\section{References}

1. https:// ssd.jpl.nasa.gov/ ?ephemerides

2. https:// naif.jpl.nasa.gov/pub/naif/ toolkit docs/ C/ req/ frames.htm I\#Selecting a Name

3. P. J. Westwick, Into the Black, Jale University Press, New Haven, 2007.

4. http:// trajectory.grc.nasa.gov/tools/ midas.sh tml.

* Corresponding author: giancarlo.genta@polito.it
5. A.B. Sergeyevsky, Interplanetary mission design handbook. Volume 1, part 1: Earth to Venus ballistic mission opportunities, 19912005, ntrs.nasa.gov, 1983.

6. A.B. Sergeyevsky, G.C. Snyder, R.A. Cunniff, Interplanetary mission design handbook. Volume 1, part 2: Earth to Mars ballistic mission opportunities, 1990-2005, ntrs.nasa.gov, 1983.

7. L.E. George, L.D. Kos, Interplanetary Mission Design Handbook: Earth-to-Mars Mission Opportunities and Mars-to-Earth Return Opportunities 2009-2024, ntrs.nasa.gov, 1998.

8. L.M. Burke, Interplanetary Mission Design Handbook: Earth-to-Mars Mission Opportunities 2026 to 2045, ntrs.nasa.gov, 2010.

9. S. Kemble, Interplanetary mission analysis and design, Springer Science \& Business Media, 2006.

10. G. R. Hintz, Orbital mechanics and astrodynamics: techniques and tools for space missions. Springer, 2015.

11. A. Shefer, New method of Orbit Determination from Two Position Vectors Based on Solving Gauss's Equations, Solar System Research, Vol. 44, No. 3, pp. 252-266.

12. http:// ssd.jpl.nasa.gov/ ?planet_pos.

13. E.Y. Choueiri, A critical history of electric propulsion: the frist 50 years (1906-1956), Journal of propulsion and power, vol 20, pp 193-203, 2004. 
14. R.G. Jahn, E.Y. Choueiri, Electric propulsion, Encyclopedia of physical science and technology, New York: Academic press, 2002

15. F. Chang Diaz, E. Seedhouse, To Mars and Beyond, Fast!: How Plasma Propulsion Will Revolutionize Space Exploration, Springer Praxis Books, New York, 2017

16. G. Genta, Next Stop Mars: The Why, How, and When of Human Missions, Springer Praxis, New York, 2017.

17. J.S. Clark, J.A. George, L. Gefert, M. Doherty, R. Sefcik, Nuclear electric propulsion: A better, safer, cheaper transportation system for human exploration of Mars, NASA tecnical memoandum 06406, 1994

18. R. C. Woolley, A. K. Nicholas, SEP Mission Design Space for Mars Orbiters, AAS/AIAA Astrodynamics Specialist Conference, Vail, Colorado, Aug.. 2015

19. J. Z. Ben-Asher. Optimal Control Theory with Aerospace Applications, AIAA Education Series. Reston, VA, USA: American Institute of Aeronautics and Astronautics, 2010. isbn: 978-1600867323.

20. J.P. Marec, Optimal Space Trajectories, Elsevier, New York, 1979.

21. D. B. Langmuir, Low-Thrust Flight. Constant exhaust velocity in Field-Free Space, in Space Technology, H. Seifert, Ed. (John Wiley and Sons, Inc., New York, 1959), Chap. 9.

22. T.M. Edelbaum, Optimal Space Trajectories, Analytical Mechanics Associates, Jericho, 1969.

23. J.H. Irving, Low-Thrust Flight. Variable exhaust velocity in Gravitational Fields, in Space Technology, H. Seifert, Ed. (John Wiley and Sons, Inc., New York, 1959), Chap. 10.

24. C. Circi Mars and Mercury missions using solar sails and solar electric propulsion, Journal of Guidance, Control, and Dynamics, Vol. 27(3), 2004, pp. 496--498.

25. S. N. Williams and V. L. Coverstone-Carroll Benefits of solar electric propulsion for the next generation of planetary exploration missions, The Journal of the Astronautical Sciences, Vol. 45(2), 1997, pp. 143--160.

26. M. Kim, Continuous Low-Thrust Trajectory Optimization: Techniques and Applications, Virginia Polytechnic Institute and State University,Blacksburg, Virginia, 2005.

27. G. Genta, P. F. Maffione, Low Thrust Interplanetary Transfers: Second Approximation Computation of Planetocentric Phases, Advances in Aerospace Science and Technology, 2017,

28. P.W. Keaton, Low Thrust Rocket Trajectories, LA10625-MS, Los Alamos, 2002.

29. G. Genta, P. F. Maffione, Optimal Low-Thrust Trajectories for Nuclear and Solar Electric Propulsion, Acta Astronautica, Vol. 118, p. 251-261, 2016.

30. http://www.adastrarocket.com/aarc/VASIMR.

31. http://www.nasa.gov/home/hqnews/2008/dec/HQ_08332 VASIMR engine.html.

32. L.F Shampine, M.W. Reichelt, and J. Kierzenka, Solving Boundary Value Problems for Ordinary Differential Equations in MATLAB with bvp4c, http://www.mathworks.com/bvp_tutorial.

33. M. Rieck, M. Bittner, B. Gruter, and J. Diepolder. FALCON.m User Guide. Institute of Flight System Dynamics, Technical University of Munich, 2016. url: www.falcon-m.com.

34. A. Wachter and L. T. Biegler. On the Implementation of a Primal-Dual Interior Point Filter Line Search Algorithm for Large-Scale Nonlinear Programming. In: Mathematical Programming Vol. 106. No. 1 (2006)
35. J. T. Betts. Practical Methods for Optimal Control and Estimation Using Nonlinear Programming. Second edition, Advances in Design and Control. Philadelphia: SIAM, Society for Industrial and Applied Mathematics, 2009.

36. F. Topputo, C. Zhang. Survey of direct transcription for low-thrust space trajectory optimization with applications. In Abstract and Applied Analysis (Vol. 2014). Hindawi Publishing Corporation.

37. H. White, P. March, et al. Measurement of Impulsive Thrust from a Closed RadioFrequency Cavity in Vacuum, Journal of Propulsion and Power, 1, 12, 2016.

38. G. Matloff, The starflight handbook, Wiley, New York, 1989.

39. https://breakthroughinitiatives.org/initiative/3

40. G. Genta, P. F. Maffione, Fast Human Mars Missions: what are the actual requirements, 10th IAA Symposium on the Future of Space Exploration: Towards the Moon Village and Beyond, Torino, June 2017.

41. G. Genta, P. F. Maffione, A graphical tool to design two-ways human Mars missions, Acta Astronautica, to be published 